\title{
Über die Gewebsatmung und Quellbarkeit des Bluts beim Ikterus.
}

\author{
Von \\ Eishichiro Tsukamoto. \\ (塚 本 策 七 郎) \\ (Aus der medizinischen Klinit con Prof. Dr. T. Kato \\ an der Tohoku Universitat su Sendai.)
}

Bekanntlich ist die Leber ein Organ, in welchem mannigfaltige wichtige Vorgänge, Oxydation, Reduktion, Spaltung und Synthese, sich abspielen; irgendeine Funktionsstörung ruft auffallende Änderung verschiedener Lebensprozesse hervor. In hiesigen Laboratorium wies Odaira ${ }^{1)}$ an Kaninchen nach, dass beim experimentell durch Unterbindung des Gallenwegs erzeugten Obstruktionsikterus der Grundumsatz herabgesetzt wird; Y a maguchi, Kimura, Tominaga und A be, dass beim Ikterus der Wasseraustansch des Organismus sowie der Sauerstoffzehrung der Blutzellen erheblich verzügert wird. In vorliegender Untersuchnug habe ich ermittelt, welehen Einfluss dor durch experimentellen Stanungsikterus bedingte cholämische Zustand auf' den Stoff- bzw. Energiewechsel der Organzellen und Erythrozyten sowie auf das Quellungsvermögen letaterer ausïbt.

Untersuchungsmethode: Unter aseptischen Kautelen unterbindet man den Ductus choldochus des gesunden Kaninchens mit einem dicken Seidentiden. Bei den Tieren zeigt sich schon den Tag darauf Ikterus. Drei bis sechs Tage nach der Operation, nach deutlichem Auftreten von Ikterus, wird dem Kaninchen ans A. carotis Blut aseptisch entnommen, dies wird defibriniert und anter Schüttehn mit Sanerstoff gesättigt. Eine Probe des Bluts wird sofort mittels eines Hämatokrits auf sein Ery throzyten-

1) Odaira, Tohoku Journ. Exp. Med., 1926, 7, 106.

2) Yamaguchi, Kimura, Tominaga u. Abe, Nippon-Naika-Gakkaj-Zasshi, $1928,15,733$. 
volum bestimmt. Der übrige, grössere Teil wird, wie in meiner früheren Mitteilung"s) ausführlich berichtet, in mehrere Atmungsgef üsse getan und in einem auf $37^{\circ} \mathrm{C}$ gehaltenen Wasserbad, welches in einem elektrischen Brutschrank steht, aufbewahrt. Nun werden die Blutproben alle drei Stunden auf den Sauerstoffverbrauch des Bluts mittelst des Barcroftschen Apparates) und zugleich auch auf die Quellbarkeit der Erythrozyten durch Messung ihres Volums mittels Hämatokrits untersucht. Von der Leber, Niere und dem Muskel desselben Tieres werden aseptisch mit einem Rasiermesser dünne Schnittstücke abgeschnitten, deren Dicke diinner als $1 \mathrm{~mm}$ und deren Gewicht unter $100 \mathrm{mg}$ sein kann, in einen auf $37^{\circ} \mathrm{C}$ gehaltenen Thermostat gebracht und nach Warburgscher Methode ${ }^{536)}$ auf den Sauerstoffverbrauch der Schnitte gemessen. Das Gefäss, in welchem die Schnittstücke enthalten sind, wird 80 oder $90 \mathrm{mal}$ pro Minute in dem Wasserbad geschüttelt; die Manometerskala des Ge-

3) Tsukamoto, Tohoku Journ. Exp. Med., 1925, 6, 286.

4) Barcroft, The respiratory funktion of the blood. Cambridge 1914, 290.

5) Warburg, Abderhalden: Handbuch d. biolog. Arbeitsmethoden. IV, Berlin und Wien 1923, 251.

6) Warburg, Biochem. Zeitschr., 1923, 142, 317.

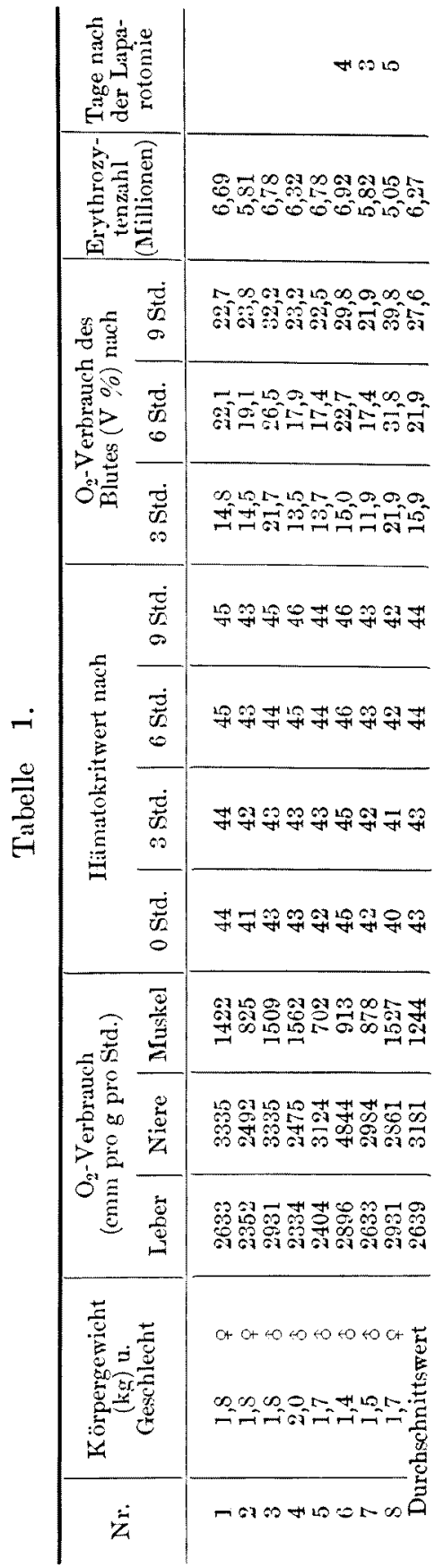




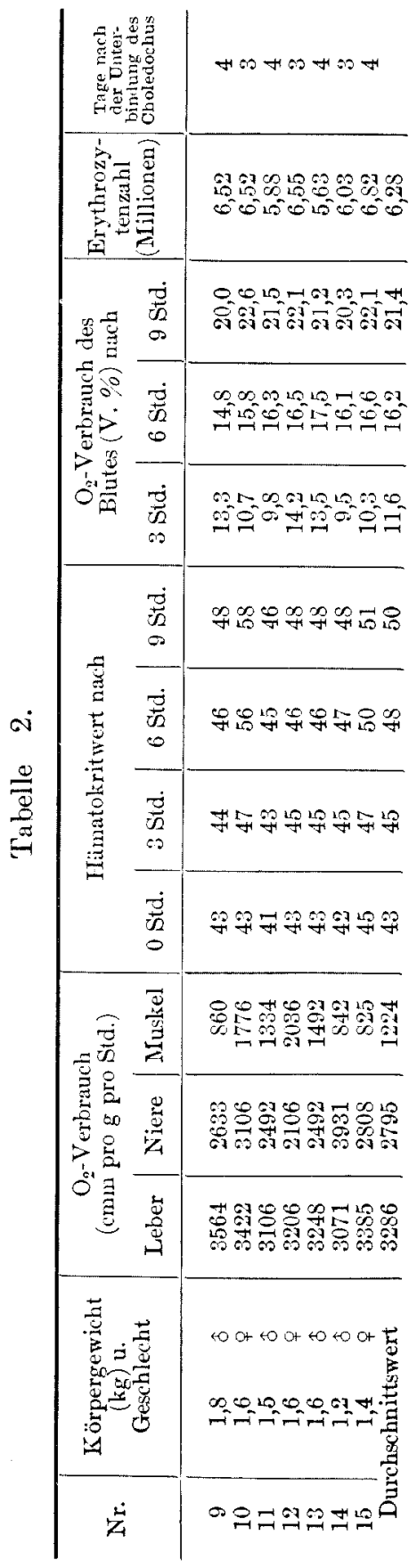

fässes meistens alle zehn oder zwanzig Minuten abgelesen. Auf diese Weise beobachtete ich 60 bis 120 Minuten lang den Verlauf des Sauerstoffverbrauchs* der verschiedenen Gewebe. Als Kontrollversuche dienten die Gewebe und das Blut von gesunden intakten Kaninchen sowie nur laparotomierten ohne Unterbindung des Ductus choledochus. 'Tab. 1 zeigt die Daten der Kontrollversuche und Tab. 2 die der Versuche an cholämischen Kaninchen. Vergleicht man die beiden Tabellen, so ist leicht zu ersehen, dass der Sauerstoffverbrauch der Niere, des Muskels und des Bluts beim Ikterus auffallend kleiner als der der Kontrolle ist, aber der der Leber gerade umgekehrt, ferner dass die Quellbarkeit der Erythrozyten beim Ikterus weit grösser ist als in der Norm.

Es ist schon von Yamaguchi, Kimura u. a. ${ }^{2)}$ darauf hingewiesen, dass sich der Sauerstoffverbrauch des Blutes beim Ikterus vermindert, was ich auch in den vorliegenden Versuchen konstatiert habe. Yamaguchi $\mathrm{i}^{(8)}$

*) Die Zahlen des Sauerstoffverbrauchs der Gewebe in allen folgenden Tabellen geben $\mathrm{cmm}$ pro $\mathrm{g}$ pro Stunde wieder.

7) Yamaguchi, Tohoku Journ. Exp. Med., 1927, 9, 501.

8) Yamaguchi, Tohoku Journ. Exp. Med, 1927, 9, 551. 
hat sich dahin geäussert, dass bei einigen Nierenleiden und schweren Beriberikranken das Gewebe in seiner Affinität für den Sauerstoff herabgesetzt ist und infolge der mangelhaften Aufnahme des Sauertoff's unter gewissen Bedingungen leichter dem asphyktischen Zustand anheimfällt, was zu vermehrtem Quellungsvermögen des Gewebes führt. Denselben Befund hatte Y a magu chi $i^{2}$ auch beim Ikterus. Sehr wahrscheinlich hat auch das Blut des ikterischen Kaninchens, welches man als flüssiges Gewebe ansehen darf, eine herabgesetzte Affinität für den Sauerstoff; infolgedessen wird das Quellungsvermögen der Blutzellen unter der unvollständigen Verbreunung allmählich gesteigert. Gleich wie im ikterischen Blut ist die Zellatmung im ikterischen Nieren- und Muskelgewebe erheblich herabgesetzt. Umgekehrt ist aber die Sachlage beim ikterischen Lebergewebe, wo sich ganz veränderte Vorgänge abspielen. Dass unter pathologischen Bedingungen die Leber, ein Organ, welches den Ofen für verschiedene lebhafte chemische Vorgänge bildet, im Energieumsatz sich anders verhält, ist nicht schwer zu vermuten.

Beim Fieber ist mach $\mathrm{Krch} I$ und $\mathrm{Kratsch},{ }^{9 /} \mathrm{Hirsch}, \mathrm{M}$ üller und $R_{0} l_{l y}{ }^{10}$ die Temperatur der Leber wärmer als die der anderen Organe oder des arteriellen Blutes. Bauer und Spiege $\mathrm{l}^{12}$ und Fisch le $\mathrm{r}^{12)}$ haben nachgewiesen, dass bei Unterbindung des Ductus choledochus die Leberzellen des zentralen Acinus durch Gallenstauung mechanisch gereizt und geschädigt werden, dadurch schnell der Autolyse unterliegen und dann freie Energie bilden können. Bei dem durch Unterbindung des Ductus choledochus bedingten Obstruktionsikterus wird der vitale Vorgang der Leber von der gestauten Galle nicht nur chemisch, sondern auch mechanisch-physikalisch beeinflusst, so dass der Energieumsatz des ikterischen Lebergewebes, verschieden von den anderen Organen, merklich erhöht wird.

\section{Zusammenfassung.}

1. Bei dem durch Unterbindung des Ductus choledochus erzeugten Obstruktionsikterus wird der Sauerstoffverbrauch des Bluts erniedrigt und die Quellbarkeit der Erythrozyten gesteigert.

9) Krehl u. Kratsch, Arch f. exp. Path. u. Pharm., 1898, 41, 185.

10) Hirsch, Müller u. Rolly, Deutsch. Arch. f. klin. Med., 1903, 75, 264.

11) Bauer u. Spiegel, Deutsch. Arch. f. klin. Med., 1919, 129, 17.

12) Fischler, Physiologie u. Pathologie d. Leber, Berlin 1925, 197. 
2. Beim Obstruktionsikterus wird die Zellatmung der Niere und des Muskels herabgesetzt, hingegen die der Leber erheblich gesteigert. 